\title{
Anabases
}

\section{D’un Ovide chrétien à un Ovide burlesque, du Moyen Âge au Grand Siècle : continuités et changements dans la traduction et dans l'illustration des Métamorphoses perçus à travers deux éditions du XVII ${ }^{\mathrm{e}}$ siècle}

\section{Basil Nelis}

\section{OpenEdition}

\section{Journals}

Édition électronique

URL : https://journals.openedition.org/anabases/10008

DOI : 10.4000/anabases. 10008

ISSN : 2256-9421

Éditeur

E.R.A.S.M.E.

Édition imprimée

Date de publication : 21 octobre 2019

Pagination : 143-160

ISSN : 1774-4296

Référence électronique

Basil Nelis, «D'un Ovide chrétien à un Ovide burlesque, du Moyen Âge au Grand Siècle : continuités et changements dans la traduction et dans l'illustration des Métamorphoses perçus à travers deux éditions du xvı" siècle », Anabases [En ligne], 30 | 2019, mis en ligne le 21 octobre 2021, consulté le 08 novembre 2021. URL : http://journals.openedition.org/anabases/10008 ; DOI : https://doi.org/ 10.4000/anabases.10008 

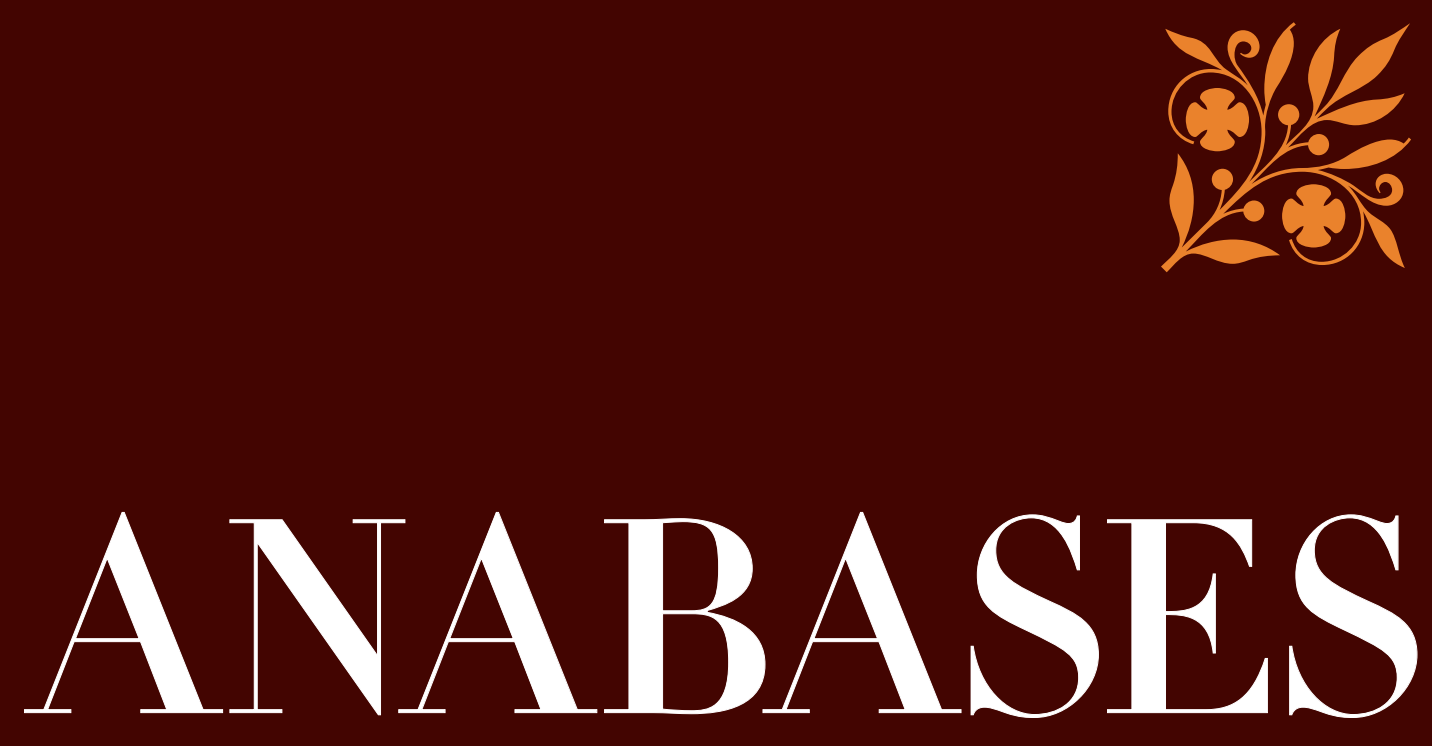

Traditions et Réceptions de l'Antiquité

\section{$\mathrm{N} \times 30$ \\ 2019}

Le sanctuaire de Gournay-sur-Aronde Le pindarisme et l'archéologie musicale Hélène en Égypte Perpétuer Ovide aux $X I V^{e}-X V I I I^{e}$ siècles II classico si fa pop Relire Marcel Detienne Freud à Pompéi 
ANABASES

Traditions et Réceptions de l'Antiquité

Revue de l'équipe de recherche E.R.A.S.M.E.

Université Toulouse-Jean Jaurès (UT2J)

Anabases dispose d'un Comité de lecture international. Chaque article envoyé à la rédaction est soumis, une fois anonymisé, à l'expertise de deux spécialistes qui rendent un rapport écrit. Les deux rapports anonymisés sont transmis à l'auteur qui tient compte des observations en vue de la publication.

\section{Comité SCIENTIFIQue}

Germaine Aujac (université Toulouse-Jean Jaurès : histoire de la géographie et des sciences antiques)

Florence Bouchet (université Toulouse-Jean Jaurès : littérature médiévale)

Hinnerk Brunns (CNRS : histoire économique et sociale ancienne et contemporaine)

Paulo Butti de Lima (université de Bari : historiographie et réception de l'Antiquité)

Luciano CANFora (université de Bari : littérature et histoire anciennes, historiographie)

Giovanna Ceserani (Stanford University : histoire intellectuelle et historiographie de la tradition classique)

Temístocles Cezar (université de Porto Alegre : historiographie moderne)

Serafina Сuомо (University of London, Birkbeck College : histoire des mathématiques et des sciences)

Paul Demont (université de Paris Sorbonne : philologie grecque et héritage classique)

Marie-Laurence Desclos (université de Grenoble II : philosophie de l'Antiquité)

Olivier Devillers (université de Bordeaux 3 - Michel-de-Montaigne : littérature et historiographie latines)

Andrea Giardina (Istituto italiano di scienze umane : histoire du monde romain et de ses réceptions)

Ève Gran-Aymerich (AIBL : histoire de l'archéologie et des transferts culturels)

François Hartog (eHess : historiographie ancienne et moderne)

Geneviève Hoffmann (université de Picardie : histoire des mondes grecs)

Christian JACOB (CNRS/EHESS : histoire comparée et épistémologie des savoirs)

Suzanne Marchand (Louisiana State University : histoire du classicisme et de l'orientalisme)

Wilfried NIPPEL (Humboldt Universität Berlin : histoire et historiographie de l'Antiquité)

Sylvie Pitria (université de Paris I-Panthéon Sorbonne : histoire et historiographie du monde romain)

Stéphane Ratтi (université de Franche-Comté - Besançon : philologie et héritage latin)

Comité de RÉdACtion

Clément Bertau-Courbières, Corinne Bonnet, Laurent bricault, Clément Bur, Adeline Grand-Clément, Anne-Hélène Klinger-Dollé, Véronique Krings, Thibaud Lanfranchi, Pascal Payen, Grégory Reimond, Sarah Rey, Catherine Valenti, Noémie VillacèQue

ÉditeUr RESPONSABLE

Clément Bur

Éditrice ADJOINTE

Catherine VALENTI

Sites Web

http://plh.univ-tlse2.fr

Revues.org : http://anabases.revues.org

Aвonnement et vente aU numéro

Éditions De Boccard - 4, rue de Lanneau - 75005 Paris

info@deboccard.com - www.deboccard.com

Tél. : 0033/(0)143260037 - Fax : 0033/(0)143548583 




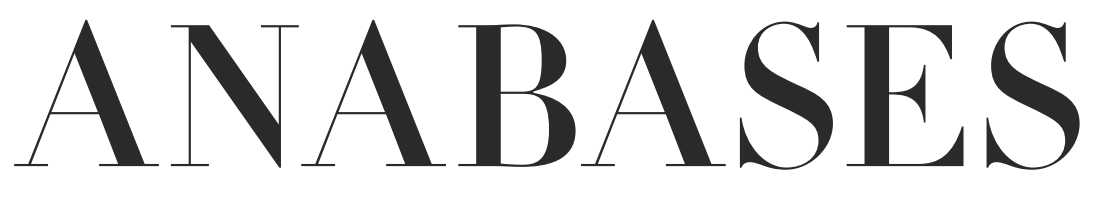

Traditions et Réceptions de l'Antiquité

$$
\begin{aligned}
& N \circ 30 \\
& 2019
\end{aligned}
$$

\section{E.R.A.S.M.E.}

Université Toulouse - Jean Jaurès 



\section{Sommaire}

ํo30 - 2019

\section{Historiographie et identités culturelles}

\section{Carole Quatrelivre}

Le sanctuaire gaulois de Gournay-sur-Aronde (Oise).

Retour sur une découverte exceptionnelle des années I970 . . . . . . . . II

\section{Traditions du patrimoine antique}

Christophe Corbier

Le pindarisme et l'archéologie musicale : style, valeur et authenticité

de la première Pythique à l'époque moderne . . . . . . . . . . . . . . .

Arnaud AmiLIEN

Hélène en Égypte : Hérodote en dialogue avec l’épopée . . . . . . . . . .

\section{Archéologie des savoirs}

Sébastien Cazalas

Au jardin des exempla. Rhétorique et stratégie de l'exemplum antique

dans l'œuvre politique de Jean Juvénal des Ursins (I388-ı473) . . . . . . 7 7

Dossier - Perpétuer Ovide : aspects moraux, éditoriaux,

linguistiques et culturels $\left(\mathrm{XIV}^{\mathrm{e}}-\mathrm{XVIII}^{\mathrm{e}} \mathrm{s}\right.$.)

Francesca Dell'Oro

Introduction $\ldots \ldots \ldots \ldots$. . . . . . . . . . . . . . 89

Hélène Casanova-Robin

L’audace châtiée : Phaéton, Actéon et Icare dans la tradition latine jusqu'à la Renaissance, tours et détours d'un symbolisme . . . . . . . . 9 93 
Dylan Bovet

Le commentaire latin des Métamorphoses d'Ovide : pratiques humanistes et évolutions de Regius-Micyllus (I543) à Burmann-Heinsius (I727) . . . . III

Martine FurNo

Ovide en classe, ou un auteur en éclats . . . . . . . . . . . . . I27

Basil NELIS

D’un Ovide chrétien à un Ovide burlesque, du Moyen Âge au Grand Siècle : continuités et changements dans la traduction et dans l'illustration des Métamorphoses perçus à travers deux éditions du xvII ${ }^{\mathrm{e}}$ siècle . . . . . . . I I43

Olivier ThÉvenAz

Épilogue ....................... I6

\section{Actualités et débats}

Tiphaine-Annabelle BEsnard

2019 : l'année pop des musées d'archéologie. Retour sur l'exposition

romaine Il classico sifa pop. Di scavi, copie e altripasticci . . . . . . . . . . I I7I

\section{Lire, relire la bibliothèque des sciences de l'Antiquité}

Jean-Pierre Albert

Le premier Detienne : une relecture de

“ La notion mythique d"A $\lambda \hat{n} \theta \varepsilon 1 \alpha$ » $\left(R E G\right.$, I96o, p. 27-35) . . . . . . . . . . $\quad{ }_{779}$

Marcel Detienne

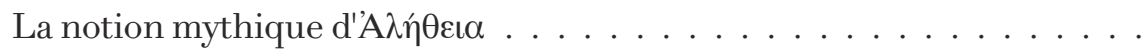

\section{Ateliers de l'histoire}

Antiquités numériques (coordonné par Elodie Guillon) ( $n^{\circ} 1$ )

Élodie GuILlon

Introduction

Jaime Alvar

Le projet EPIDI : Epítetos divinos.

Experiencia religiosa y relaciones de poder en Hispania . . . . . . . . . . . I98 Les mots de l'Antiquité (coordonné par Magali Soulatges) ( $n^{\circ} 10$ )

Jack Thомаs

L’Antiquité dans les toponymes de l'État de New York . . . . . . . . . . . 202

Actualité du théâtre (coordonné par Malika Bastin-Hammou) ( $\left.n^{\circ} 4\right)$

Mathieu FERrand

“ Avons-nous perdu le Soleil ? / Ou l'avons-nous chassé ? » 
Thyeste de Sénèque, traduit par Florence Dupont.

Mise en scène de Thomas Jolly (Avignon, 20I8) . . . . . . . . . . . . . 2 2I4

Voyages et Voyageurs (coordonné par Véronique Krings) ( $n^{\circ} 11$ )

Claude AzIzA

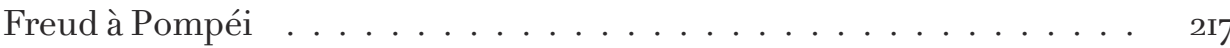

\section{Comptes rendus}

Lucile Arnoux-Farnoux et Polina Kosmadaki (dir.)

Le double voyage : Paris-Athènes (Igrg-Ig39) (Catherine Valenti) . . . . . . 225

Sandra Boehringer et Daniele Lorenzini (dir.)

Foucault, la sexualité, l'Antiquité (Jan Nelis) . . . . . . . . . . . . . . . . 226

Corinne Bonnet, Nicole Belayche, Marlène Albert Llorca,

Alexis Avdeeff, Francesco Massa, Iwo Slobodzianex (dir.)

Puissances divines à l'épreuve du comparatisme. Constructions,

variations et réseaux relationnels (Geneviève Hoffmann) . . . . . . . . . . 228

Shane ButLer (éd.)

Deep Classics, Rethinking Classical Reception (Jan Nelis) . . . . . . . . . . 23o

Zeynep ÇELIK

About Antiquities: Politics of Archaeology in the Ottoman Empire (Jorge Elices Ocón) . . . . . . . . . . . . . . . . . 231

Xavier Deru et Germaine Leman-Delerive (éd),

Franz Cumont, Comment la Belgique fut romanisée (Vivien Barrière) . . . 234

Olivier Devillers, Breno Battistin Sebastiani (éd.)

Sources et modèles des historiens anciens (Arnaud Saura-Ziegelmeyer) . . $\quad 235$

Mara Fazio, Pierre Frantz et Vincenzo De Santis (dir.)

Les Arts du spectacle et la référence antique dans le théâtre

européen (I760-I830) (Arnaud Saura-Ziegelmeyer) . . . . . . . . . . . . . . 237

Jérémy Guedu et Barbara Meazzi (dir.)

La culture fasciste entre latinité et méditerranéité (I880-1940), in Cahiers de la Méditerranée 95, (Andrea Avalli) . . . . . . . . . . . . . . . 239

Marie-Laurence HAAck (éd.), avec la collaboration de Martin MiLler, Les Étrusques au temps du fascisme et du nazisme (Jan Nelis) . . . . . . . . . 24I

Arlene Holmes-Henderson, Steven Hunt et Mai Musié (éd.)

Forward with Classics. Classical Languages in Schools and Communities

(Charlotte Tournier) . . . . . . . . . . . . . . . . . . . . . . .

Daniel Jew, Robin Osborne et Michael Scotт (éd.)

M. I. Finley. An Ancient Historian and his Impact (Hinnerk Bruhns) . . . . $\quad 244$ 
Kostas Kalimtzis,

An inquiry into the philosophical concept of scholê.

Leisure as a Political End (Florent Rouzade) . . . . . . . . . . . . . . . . . 246

Anne-Hélène KLINGER-Dollé

Le De sensu de Charles de Bovelles. Conception philosophique

des sens et figuration de la pensée. Suivi du texte latin du De sensu,

traduit et annoté (Laure Hermand-Schebat) . . . . . . . . . . . . . 248

Egidia Occhipinti

The Hellenica Oxyrhynchia and Historiography:

New Research Perspectives (Anne de Cremoux) . . . . . . . . . . . . . . . . 249

Laurent OLIVIER (dir.)

La mémoire et le temps. L'ouvre transdisciplinaire

d'Henri Hubert (I872-I927) (Sarah Rey) . . . . . . . . . . . . . . . 25I

Gabriella Pinonti et Corinne Bonnet (dir.),

Les dieux d'Homère. Polythéisme et poésie en Grèce ancienne,

Kernos, Supplément 3r. (Sandya Sistac) . . . . . . . . . . . . . . . 253

Rabun TAYLoR, Katherine W. Rinne et S. Kostof

Rome. An Urban History from Antiquity to the Present

(Cyrielle Landrea) . . . . . . . . . . . . . . . . . . . 255

Wyger Velema et Arthur Weststeisn (éd.)

Ancient Models in the Early Modern Republican Imagination

(Paulo Butti de Lima) . . . . . . . . . . . . . . . . . . . 256

Philip WaLsh (éd.)

Brill's Companion to the Reception of Aristophanes

(Malika Bastin-Hammou) . . . . . . . . . . . . . . .

Richard WARren

Art Nouveau and the Classical Tradition (Lucien Calvié) . . . . . . . . . 26o

Jesse Weiner, Benjamin Eldon Stevens et Brett M. Rogers (éd.)

Frankenstein and Its Classics. The Modern Prometheus

from Antiquity to Science Fiction (Mathieu Scapin) . . . . . . . . . . . . . 26r

Nigel G. WiLson

From Byzantium to Italy. Greek Studies in the Italian Renaissance,

deuxième édition (Luigi-Alberto Sanchi) . . . . . . . . . . . . . . 262

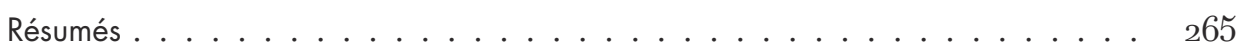

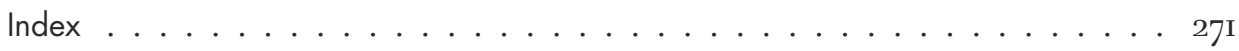




\section{Dossier dirigé par Francesca Dell'Oro et Olivier Thévenaz}

Perpétuer Ovide : aspects moraux, éditoriaux, linguistiques et culturels $\left(\mathrm{XIV}^{\mathrm{e}}-\mathrm{XVIII}{ }^{\mathrm{e}} \mathrm{s}\right.$.) 

Anabases 30 (2019), p. 143-160.

\section{D'un Ovide chrétien à un Ovide burlesque, du Moyen Âge au Grand Siècle : continuités et changements dans la traduction et dans l'illustration des Métamorphoses perçus à travers deux éditions du XVII ${ }^{\mathrm{e}}$ siècle ${ }^{1}$}

Basil NELIS

e XVII siècle marque un tournant important dans la réception des Métamorphoses en France. Un changement de paradigme lié à la traduction d'Ovide prend forme au fil du siècle, dans le cadre duquel on peut distinguer trois phases: les traductions relativement conservatrices des années 1620, les “ traductions » burlesques des années 1650 et le courant galant dans le dernier tiers du siècle ${ }^{2}$. Il s'agit, dans cette étude, d'illustrer ce changement de paradigme en se fondant principalement sur deux traductions fort différentes. La première est une

1 Cette contribution prend son origine d'un travail de Maîtrise universitaire effectuée à l'Université de Lausanne dans le cadre du programme de spécialisation en Histoire du livre et édition critique des textes. J'ai eu l'occasion d'étudier des livres anciens de la Bibliothèque Cantonale et Universitaire de Lausanne liés à la postérité d'Ovide. Il s'agissait de valoriser la collection de la Bibliothèque, et les éditions auxquelles j'ai pu avoir accès, parmi lesquelles figurent la traduction de Renouard et l'Ovide bouffon de Richer, ont dans une certaine mesure conditionné mon approche dans cet article. Mon travail a été dirigé par Francesca Dell'Oro et Olivier Thévenaz, que je souhaite remercier ici. J'aimerais également exprimer mes remerciements les plus sincères aux deux experts de la revue Anabases, dont les commentaires m'ont été d'une grande utilité et ont considérablement enrichi cette étude.

2 H. TaYlor, The Lives of Ovid in Seventeenth-Century French Culture, Oxford, 2017, p. 17. 
édition illustrée de la traduction des Métamorphoses par Nicolas Renouard (1617), qui s'inscrit dans la lignée des interprétations humanistes d'Ovide du xvI siècle ; la seconde, l'Ovide bouffon (1649), est une traduction burlesque (sans illustrations) des cinq premiers livres de ce même poème ovidien par Louis Richer, un auteur dont on ne sait quasiment rien ${ }^{3}$. Ces deux éditions démontrent à quel point la manière de lire, d'interpréter et de traduire les Métamorphoses a changé en à peine quarante ans dans la première moitié du xviI siècle. Je considérerai également ces éditions dans un contexte historique plus large, dans la mesure où elles témoignent encore, à certains égards, des courants de réception antérieurs au XVII ${ }^{\mathrm{e}}$ siècle, notamment chrétiennes, qui ont fortement marqué la lecture d'Ovide en France. C'est pourquoi, dans mon analyse de l'édition de Renouard, j'accorderai un intérêt particulier au rapport entre le texte et l'image. Car la traduction, parue en 1606, est enrichie dès 1617 de gravures dans lesquelles on peut percevoir des vestiges d'une tradition illustrative dont les origines artistiques remontent aux manuscrits de l'Ovide moralisé. Pour pleinement saisir la richesse des illustrations dans l'édition de Renouard, il importe, dans un premier temps, de remonter à ce poème de 72'000 vers d'un auteur inconnu qui est l'un des textes les plus importants de l'histoire de la réception des Métamorphoses ${ }^{4}$. Les illustrations du mythe de Pyrame et Thisbé évoquent particulièrement bien la manière dont l'édition de Renouard renouvelle une tradition artistique qui remonte aux enluminures médiévales. Or, cet héritage chrétien d'Ovide, qui se perçoit chez Renouard uniquement dans les illustrations, ne correspond pas à la tonalité générale de l'édition, qui s'inscrit dans la lignée des lectures humanistes et scolastiques du xvi ${ }^{\mathrm{e}}$ siècle. En réalité, en y regardant de plus près, on verra que certains aspects du texte de Renouard semblent annoncer le changement de paradigme qui marque la traduction d'Ovide au milieu du xvis ${ }^{\mathrm{e}}$ siècle et dont l'Ovide bouffon de Louis Richer est un bon exemple. En ce qui concerne la traduction de Richer, la version burlesque du mythe de Pyrame et Thisbé contient assurément des éléments intéressants, comme on aura l'occasion de le constater; je m'attarderai toutefois davantage sur l'épisode de Narcisse.

3 Frédéric Deloffre pense que Richer a pu être greffier ou procureur (F. Deloffre (éd.), Agréables Conférences de deux paysans de Saint-Ouen et de Montmorency. Sur les affaires du temps (1649-1651), Paris, 1961, p. 23). Plus récemment, Bénédicte Gady a avancé l'hypothèse que l'écrivain burlesque Richer a pu être confondu avec un autre Louis Richer, qui était graveur, mais trop peu d'informations sont disponibles pour qu'on puisse en être certain (B. GADY, “ Louis Richer, dessinateur, graveur et satiriste au milieu du vxi ${ }^{\text {e }}$ siècle ", in D. Cordellier, (éd.), Dessiner pour graver, graver pour dessiner. Le dessin dans la révolution de l'estampe, Paris, 2012, p. 133-150).

4 On ne sait presque rien de l'auteur de ce texte, si ce n'est qu'il est probablement issu de l'entourage de la reine Jeanne de Bourgogne. M. Possamaï-Pérez, L'Ovide moralisé. Essai d'interprétation, Paris, 2006, p. 21. 
En effet, cet épisode se démarque des autres par une intervention du narrateur qui est particulièrement évocatrice de la poétique de Richer. La désacralisation du texte ovidien opérée par le poète dans sa traduction participe d'une réflexion plus large à propos de l'autorité des auteurs antiques, et l'épisode de Narcisse illustre particulièrement bien cet aspect fondamental du burlesque de Richer. Je me propose donc de partir des manuscrits médiévaux de l'Ovide moralisé afin de présenter certaines continuités qui se retrouvent jusque dans l'édition de Renouard, et de montrer ensuite, en m'appuyant sur la traduction de Richer, les débuts du changement de paradigme dans la réception d'Ovide.

Au début du livre IV des Métamorphoses (43-166), la première fille de Minyas choisit de raconter l'histoire de Pyrame et Thisbé, deux jeunes amants séparés l'un de l'autre et enfermés par leurs parents. Un mur les sépare, mais ils parviennent à communiquer par une fissure dans celui-ci. Une nuit, ils décident de sortir pour se rencontrer. Avant l'arrivée de Pyrame, Thisbé aperçoit une lionne qui s'abreuve à une fontaine, la gueule en sang après la chasse ; terrifiée, elle réussit à s'enfuir, mais dans sa précipitation elle perd son voile que la lionne déchire et ensanglante. Lorsque Pyrame arrive, il trouve le voile de son amante et, pensant que Thisbé a été dévorée par la lionne, se suicide. Quand Thisbé arrive sur les lieux du désastre, elle voit le cadavre de Pyrame et, désespérée, se suicide à son tour. Le récit de Pyrame et Thisbé est l'un des épisodes des Métamorphoses les plus riches du point de vue de la réception : des échos ovidiens se retrouvent dans de nombreux textes au fil des siècles, parmi lesquels on peut citer la tragédie de Théophile de Viau intitulée Les Amours tragiques de Pyrame et Thisbé (1617), ou Romeo and Juliet de William Shakespeare (1597), où la présence d'Ovide est néanmoins plus diffuse $^{5}$. En réalité, ce mythe fascine déjà au xııI ${ }^{\mathrm{e}}$ siècle, et le récit est même adapté dans une version de la Bible rédigée par le clerc lorrain Jean Malkaraume ${ }^{6}$. Cet amalgame de matières profane et biblique exemplifie la réception chrétienne que connaissent les Métamorphoses au cours du Moyen Âge, et jusqu'à la Renaissance. Au début du Xıv siècle, période pendant laquelle est composé l'Ovide moralisé, un moine bénédictin ami de Pétrarque, Pierre Bersuire, écrit un Ouidius moralizatus,

5 Si l'on retrouve certaines allusions à Ovide dans la tragédie de Shakespeare, la pièce du dramaturge anglais est toutefois plus directement inspirée par les récits de Luigi da Porto et de Matteo Bandello (sur cette question, voir J. Levinson, « Romeo and Juliet before Shakespeare », Studies in Philology 81, 1984, p. 325-347). Sur la présence du mythe de Pyrame et Thisbé chez Shakespeare, voir A. B. TaYlor, "Ovid's Myths and the Unsmooth Course of Love in A Midsummer Night's Dream ", in C. Martindale et A. B. TAYLor (éds.), Shakespeare and the Classics, Cambridge, 2004, p. 49-65.

6 M. Moussy, “La moralisation du mythe: Pyrame et Thisbé dans la Bible de Jean Malkaraume ", in L. Harf-Lancner (éd.), Ovide métamorphosé. Les lecteurs médiévaux d'Ovide, Paris, 2009, p. 83-103. 
texte en prose rédigé dans un latin clérical qui fournit lui aussi une interprétation allégorique des Métamorphoses ${ }^{7}$. Et ce n'est pas un hasard si une édition importante du texte datant de la fin du $x^{\mathrm{e}}$ siècle, qui n'est en réalité pas autre chose qu'une “ paraphrase de l'Ovide moralisé $)^{8}$, porte le titre évocateur de Bible des poëtes (1493).

L'Ovide moralisé est la première traduction française des Métamorphoses, et chaque " fable » est suivie d'une interprétation allégorique destinée à rendre le texte acceptable pour un public chrétien`. L'immense succès d'Ovide à partir des XII $^{\mathrm{e}}-\mathrm{XIII}^{\mathrm{e}}$ siècles, époque baptisée aetas Ouidiana par le philologue munichois Ludwig Traube ${ }^{10}$, est en partie dû au fait qu'on le considérait comme un préfigurateur du christianisme, et cette conception a longtemps conditionné la lecture des Métamorphoses, qui sont, “ avec la Bible, le livre le plus lu et le plus apprécié de tout le Moyen Âge „" ${ }^{1}$. L'auteur de l'Ovide moralisé souhaite mettre en avant l'adaptabilité de la matière ovidienne à la foi chrétienne. Tout en traduisant les fables et en respectant leur ordre, l'auteur entreprend, pour chacune d'entre elles, de l'interpréter pour en dégager ce qu'il appelle la vérité, le sens caché, soit, pour reprendre ses propres termes, de dire “ce que j'en entens » ${ }^{12}$. Ainsi, l'Ovide moralisé est à la fois une traduction française des Métamorphoses et une interprétation allégorique du texte latin. L'épisode de Pyrame et Thisbé dans

7 K. McKinley, Reading the Ovidian Heroine. Metamorphoses Commentaries 1100-1618, Leyde, 2001, p. 106-107.

8 G. Amielle, Recherches sur des traductions françaises des Métamorphoses d'Ovide illustrées et publiées en France à la fin du xv siècle et au xvI siècle, Paris, 1989, p. 9. En réalité, la Bible des poëtes se fonde plutôt sur les manuscrits de l'Ovide moralisé en prose : en effet, le succès de l'Ovide moralisé en vers est tel qu'il est réécrit en prose durant le $\mathrm{xv}^{\mathrm{e}}$ siècle. Sur l'Ovide moralisé en prose, voir L. Harf-Lancner et M. PÉrezSimon, “Une lecture profane de l'Ovide moralisé. Le manuscrit BnF français 137 : une mythologie illustrée ", Cahiers de recherches médiévales et humanistes 30 (2015), p. 167-196.

9 La division du texte des Métamorphoses en “fables ", ou argumenta, remonterait à la structure du poème opérée par le Pseudo-Lactance; sur cette division du texte, voir R. Hexter, “Medieval articulations of Ovid's Metamorphoses : from Lactantian segmentation to Arnulfian allegory », Mediaevalia 13 (1987), p. 63-82. Toutefois, la question reste débattue: voir A. Cameron, “An Anonymous Ancient Commentary on Ovid's Metamorphoses ", in Greek Mythography in the Roman World, Oxford, 2004, p. 3-32.

10 L. Traube, Vorlesungen und Abhandlungen II. Einleitung in die lateinische Philologie des Mittelalters, Munich, 1911, p. 113.

1 F. Clier-Colombani, Images et imaginaire dans l'Ovide moralisé, Paris, 2017, p. 11.

12 Ovide moralisé I, 18, avec le texte de C. Baker et alii (éds.), Ovide moralisé : Livre I, 2 vol., Paris, 2018, qui modifie ici la leçon retenue par De Boer (i.e. « ce que j’entens »). 
l'Ovide moralisé incarne un exemple particulièrement saisissant de cette dualité textuelle; après avoir rendu le sens original du texte, le traducteur en explique le sens caché, attirant l'attention sur la dimension allégorique de la fable (Ovide moralisé, IV. 1176-77) ${ }^{13}$ :

Or vous dirai l'alegorie

Que ceste fable signefie...

L'auteur cherche littéralement à dévoiler le texte, le dépouiller de son integumentum, afin d'en révéler le sens véritable (sa senefiance), correspondant à la morale chrétienne, qui est recouvert par une alegorie ${ }^{14}$. La réappropriation chrétienne du récit ovidien devient encore plus explicite un peu plus loin, lorsque les deux amants sont explicitement comparés à des figures de martyrs (Ovide moralisé, IV. 1207-10, je souligne) :

Aussi soloient jadis faire

Li saint martir, qui despisoient

Le monde et pour Dieu se livroient

A tous martires endurer.

Cette christianisation d'Ovide se remarque non seulement dans le texte, mais encore dans l'image. Les enluminures des manuscrits de l'Ovide moralisé ont bien souvent été négligées par les philologues, si bien qu'on a pu parler de “préjugé textologique ${ }^{15}$. Or, les illustrations sont également un vecteur important de l'allégorisation des fables. Dans certaines illustrations, Pyrame et Thisbé sont représentés comme des martyrs chrétiens. On est bien loin de l'imaginaire païen et d'une simple histoire d'amour malheureux. L'histoire devient celle d'un sacrifice à dimension chrétienne et Pyrame et Thisbé apparaissent comme des figures de saints. Mais il existe également des représentations plus conventionnelles de

13 Pour le livre IV, je suis le texte de C. DE Boer (éd.), Ovide moralisé : poème du commencement du quatorzième siècle, 5 vol., Paris, 1915-1938.

14 L’idée qu'il existe sous la fable une vérité latente à découvrir est bien exprimée au début du premier livre (Ovide moralisé I, 44-46) : Qui le sens en porroit savoir, | la veritez seroit aperte, | qui souz les fables gist couverte.

15 F. Cinato et A. Surprenant, "L'escrime scolastique du Liber de Arte dimicatoria », in Quand l'image relit le texte, Paris, 2013, (p. 249-270), p. 254. Cité dans Clier-Colombani, Images, p. 11. Récemment, les illustrations de l'Ovide moralisé ont suscité un regain d'intérêt critique, notamment avec la parution en 2015 d'un numéro des Cahiers de recherches médiévales et humanistes consacré à l'illustration des manuscrits de l'Ovide moralisé, en 2017, de l'ouvrage de Françoise Clier-Colombani, et en 2018, d'une édition critique du livre I qui publie toutes les enluminures des différents manuscrits (C. Baker, Ovide moralisé, vol. 1, p. 290-384). 


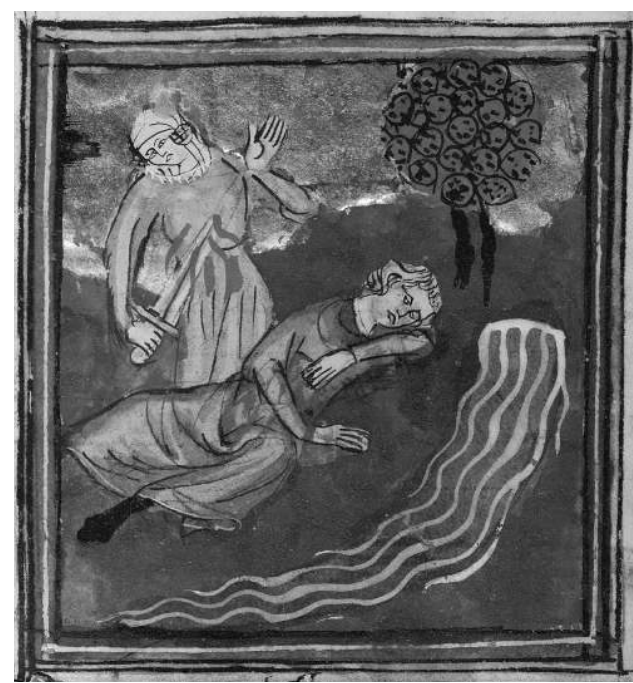

Fig. 1. Suicide de Thisbé. Source gallica. bnf.fr / Bibliothèque nationale de France (ms. Arsenal 5069, f. 42v).

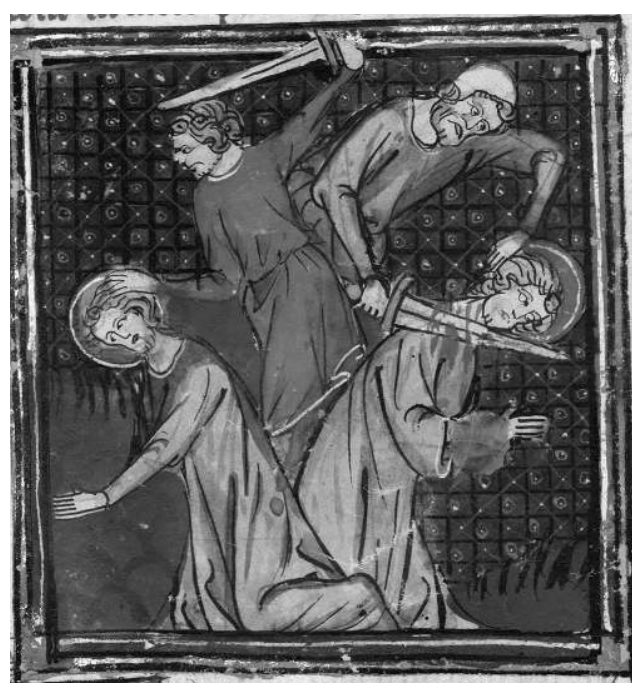

Fig. 2. Mort des amants en martyrs. Source gallica.bnf.fr / Bibliothèque nationale de

France (ms. Arsenal 5069, f. 43r).

l'univers païen des amants, et parfois les deux types d'illustrations se retrouvent sur un seul et même folio du manuscrit, comme pour symboliser la dualité de la traduction et de l'interprétation allégorique dans le texte ${ }^{16}$. Les manuscrits les plus impressionnants sont souvent les plus anciens car ils sont les plus copieusement illustrés ; le manuscrit de Rouen (ms. Bm O. 4), qui présente 453 miniatures, le manuscrit de l'Arsenal (ms. 5069), qui en compte 302, et le manuscrit de Lyon, (ms. 742) qui est orné de 57 miniatures, sont particulièrement dignes de mention ${ }^{17}$. De ces trois manuscrits, c'est celui de Rouen qui présente les plus riches illustrations, et la fable de Pyrame et Thisbé est accompagnée par une série de onze miniatures ${ }^{18}$. Dans le manuscrit de l'Arsenal comme dans celui de Rouen, Thisbé est dépeinte en train de se transpercer la poitrine avec l'épée de Pyrame qui gît devant elle, et le voile, symbole de l'erreur des amants, est clairement visible (fig. 1 et 3, enluminure de gauche). Dans les images qui christianisent la scène,

16 R. Blumenfeld-Kosinski, “ Illustrations et interprétations dans un manuscrit de l'Ovide moralisé(Arsenal 5069) ", Cahiers de recherches médiévales et humanistes 9 (2002), (p. 71-82), p. 71-73.

17 Ces trois manuscrits ont fait l'objet d'une étude par C. LoRD, « The Manuscripts of the Ovide moralisé », The Art Bulletin 57. 2 (1975), p. 161-175.

18 R. Wolf-Bonvin, “ Les Minéides au fil de leur compte. Une iconographie entre lumière et ombres (Ovide moralisé, livre IV, manuscrit Rouen, Bm. O 4) ", Cahiers de recherches médiévales et humanistes 30 (2015), (p. 75-116), p. 91-112. 


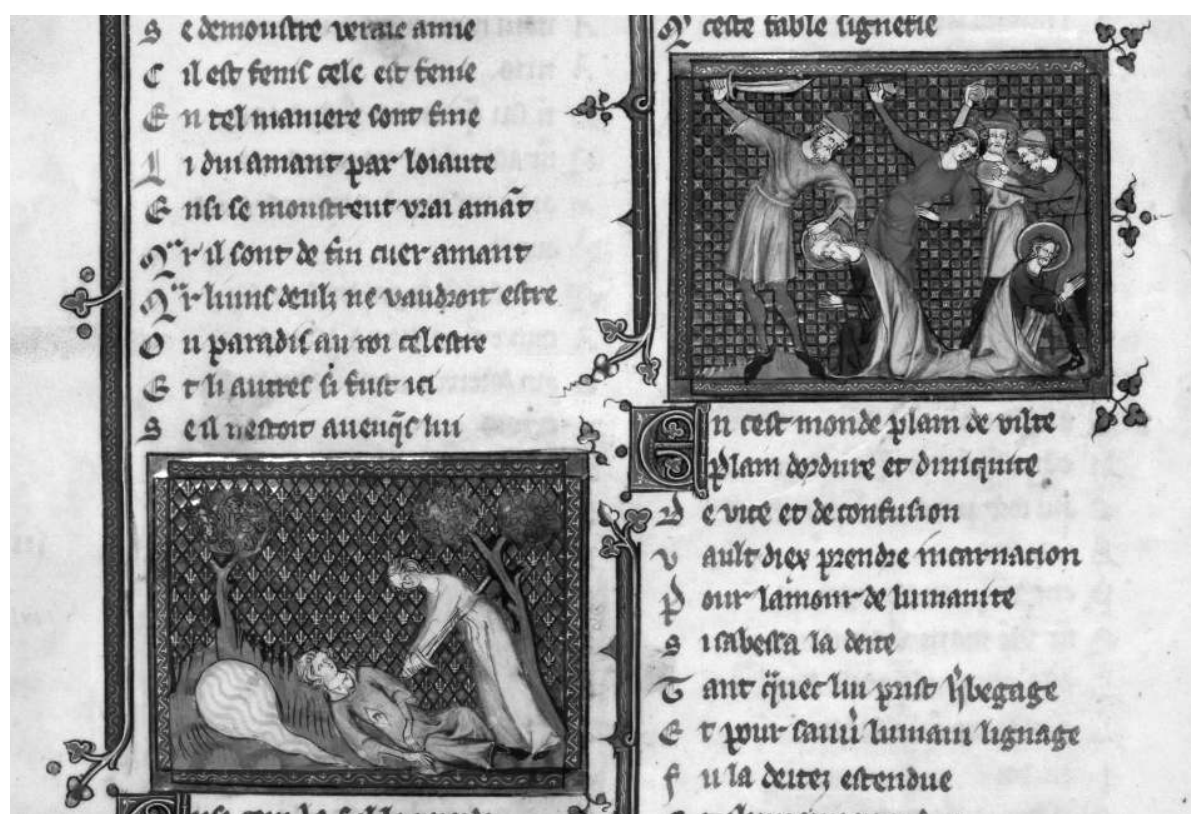

Fig. 3. Suicide de Thisbé et mort des amants en martyrs.

Bibliothèque municipale de Rouen (ms. Rouen Bm O. 4, f. 96v).

elles aussi relativement similaires dans ces deux manuscrits, les têtes auréolées de Pyrame et Thisbé sont offertes en sacrifice à des bourreaux qui s'apprêtent à les décapiter (fig. 2 et 3, enluminure de droite).

Si deux manuscrits n'offrent presque jamais exactement le même programme d'illustration, ce n'est pas le cas des livres imprimés. À la fin du xv siècle, le succès de l'Ovide moralisé est tel que des livres commencent à être imprimés à partir des manuscrits, et, dès cette période, la tradition iconographique se fixe. L'Ovide moralisé est ainsi un texte clef dans la transition du manuscrit au livre imprimé ${ }^{19}$. Dans le cas de l'illustration de la scène de Pyrame et Thisbé, on observe que la structure de l'image demeure relativement inchangée depuis les premiers incunables jusqu'à l'édition illustrée de Renouard au début du XvıI ${ }^{\mathrm{e}}$ siècle, voire encore plus tard ${ }^{20}$. Par exemple, l'incunable F5 de Lille (fig. 4) comporte des traits similaires à l'illustration du manuscrit de Rouen (fig. 3, enluminure de gauche),

19 Voir S. CERrito, “ L'Ovide moralisé à l'aube de la Renaissance. De la prose brugeoise à la Bible des poëtes », Cahiers de recherches médiévales et humanistes 30 (2015), p. 197-219.

20 Les illustrations de l'édition des Métamorphoses de Villenave (Paris, 1806-7) témoignent encore de la structure illustrative de certains manuscrits et des incunables de l'Ovide moralisé. Voir fig. 7. 


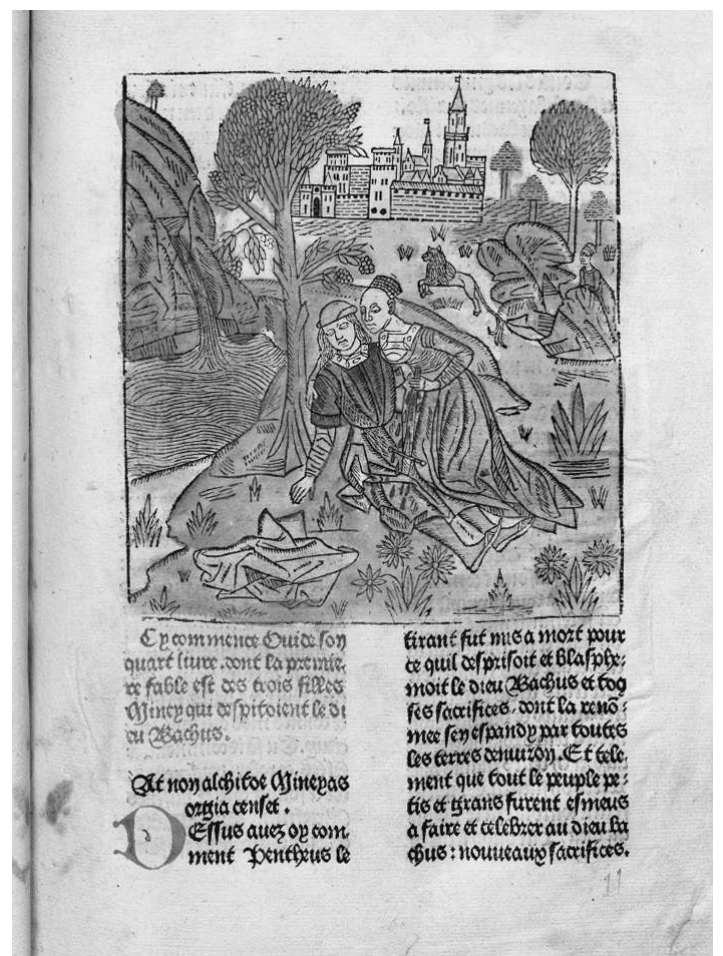

Fig. 4. Thisbé mourante, avec la lionne en arrière-plan. Bibliothèque municipale de Lille (inc F5, f. 72r). car dans les deux images Thisbé, mourante, est penchée sur le corps de son amant et son voile est clairement visible sur le sol. Ghislaine Amielle a montré que la tradition picturale liée au mythe de Pyrame et Thisbé est clairement établie depuis la parution de la Bible des poëtes en 1493 (fig. 5) ${ }^{21}$. Les illustrations de cette édition ont grandement inspiré les illustrateurs majeurs du $\mathrm{xvI}^{\mathrm{e}}$ siècle tels que Bernard Salomon et Virgile Solis, artistes centraux dans l'histoire des éditions illustrées des Métamorphoses ${ }^{22}$.

$\mathrm{Au}$ début du $\mathrm{xvII}^{\mathrm{e}}$ siècle, la réception d'Ovide est marquée par un courant d'interprétation moral des Métamorphoses. Nicolas Renouard adjoint, à la fin de sa traduction du texte qui a pu être critiquée pour son insipidité ${ }^{23}$, quinze discours contenant “ l'explication morale des fables ».

Comme à l'époque de l'Ovide moralisé, l'idée de faire coïncider le texte d'Ovide avec la bienséance du monde contemporain est toujours manifeste, mais

21 AmielLe, Recherches, p. 126.

22 Les illustrations de B. Salomon dans l'édition lyonnaise de 1557 intitulée La Métamorphose figurée ont été une source importante d'inspiration pour V. Solis, dont les illustrations paraissent à Francfort en 1563. Sur V. Solis, voir E. von Uвisch, Virgil Solis und seine biblischen Illustrationen für den Holzschnitt, Leipzig, 1989 ; sur B. Salomon, voir l'étude de P. Sharatt, Bernard Salomon, illustrateur lyonnais, Genève, 2005. Sur l'iconographie ovidienne d'une manière plus générale, voir G. HuberRebenich et alii, Ikonographisches Repertorium zu den Metamorphosen des Ovid: die textbegleitende Druckgraphik, Berlin, 2004.

23 Il s'agit, selon M. Moog-Grünewald, de « langweilige, wenig kunstvolle Prosaparaphrasen ». M. Moog-GRüNewald, Metamorphosen der Metamorphosen. Rezeptionsarten der ovidischen Verwandlungsgeschichten in Italien und Frankreich im XVI. und XVII. Jahrhundert, Heidelberg, 1979, p. 119. 


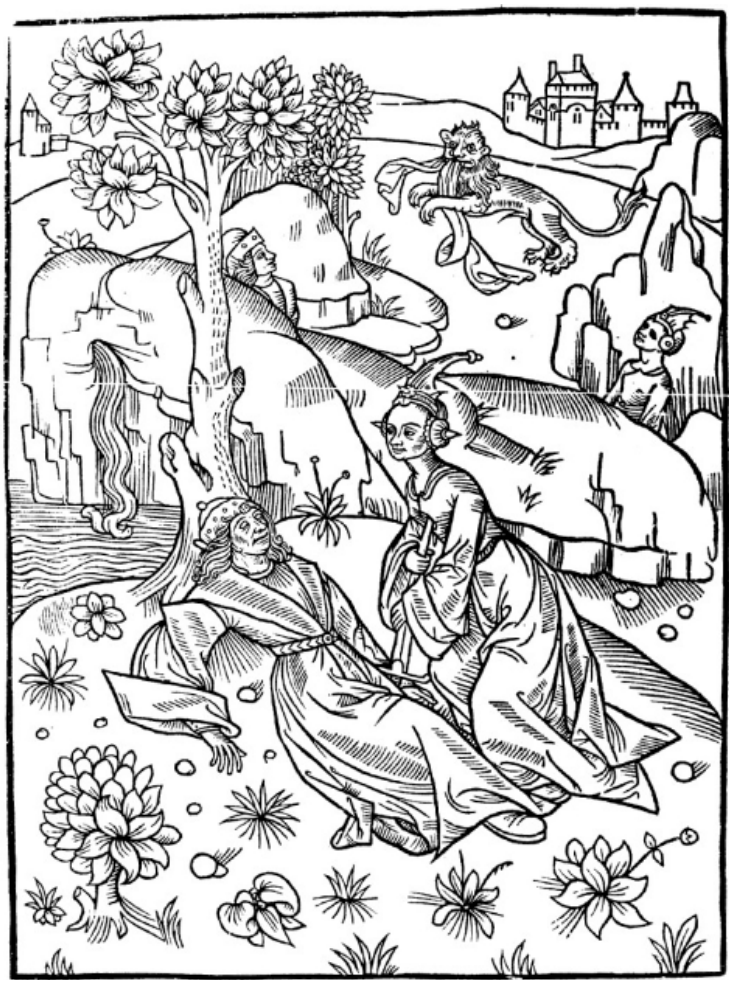

Fig. 5. Suicide de Thisbé, avec la lionne en arrièreplan. Source gallica.bnf.fr / Bibliothèque nationale de France (La Bible des poëtes, Lyon, 1493, f. 119). l'interprétation strictement chrétienne d'Ovide n'est plus d'actualité chez Renouard. Les explications de Renouard s'inspirent plutôt des commentaires humanistes, et surtout de ceux à usage scolaire comme les Mythologiae siue Explicationis fabularum libri decem de Natale Conti (1551). Une autre source très importante pour Renouard est la Fabularum Ouidii Interpretatio de Georgius Sabinus $(1555)^{24}$, qui emprunte beaucoup au commentaire des Métamorphoses de Raphaël Regius $^{25}$. Ce qui importe véritablement à Renouard, c'est de tirer une leçon morale des fables, et son interprétation est fortement marquée par le passé humaniste de l'herméneutique ovidienne. Le caractère ludique du texte d'Ovide est écarté pour laisser place à une lecture relativement austère. Pour Renouard, Pyrame et Thisbé sont des exemples d'un amour trop passionnel qui peut à terme mener à des conséquences néfastes ${ }^{26}$ :

24 Sur les sources de Renouard, voir Amielle, Recherches, p. 269 ; A. Moss, Ovid in Renaissance France. A Survey of the Latin Editions of Ovid and Commentaries Printed in France Before 1600, Leeds, 1982, p. 154 ; P.-J. SALAZAR, "Les pouvoirs de la fable: mythologie, littérature et tradition (1650-1725) ", Revue d'Histoire littéraire de France 91.6(1991), (p. 878-889), p. 881.

25 Sur le commentaire de R. Regius, voir la contribution de D. Bovet dans ce même volume.

26 N. Renouard, XV Discours contenans l'explication morale des fables, Paris, 1637 [1606], p. $50-1$. 


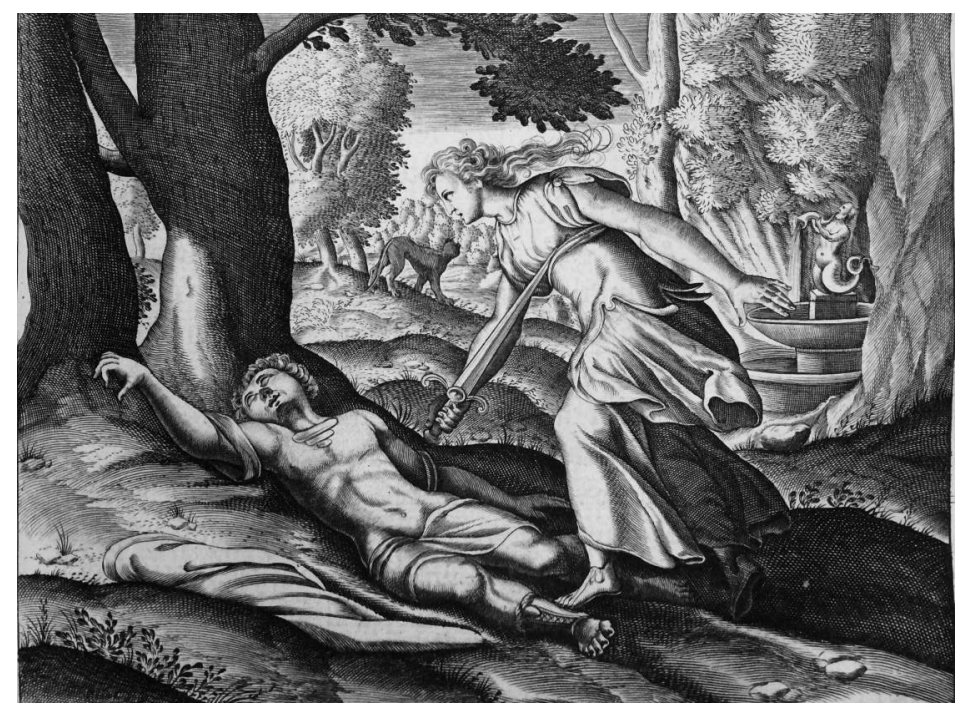

Fig. 6. Suicide de Thisbé, avec la lionne en arrière-plan.

Métamorphoses, Nicolas Renouard, Paris, 1619. Illustration : Jean Mathieu.

Bibliothèque cantonale et universitaire de Lausanne (AVB 147).

Miserables amans, falloit-il que vous fussiez consumez de si bruslantes flames, pour estre par leur violence precipitez à une si deplorable ruine, qui vous a rendus aux siecles venus en suite du vostre, rares exemples d'amour, \& ensemble tristes pourtraicts de l'infortune \& de la misere?

En parallèle, Renouard met en garde contre la sévérité des parents, qui peut également causer des situations désastreuses. Il commente en marge du texte : “L'austère sévérité des pères resistant aux affections de leurs enfans, est quelquefois dangereuse ». Mais en même temps, Renouard présente ses Discours comme une discussion entre deux hommes lettrés et raffinés qui débattent de la moralité des fables ovidiennes lors de conversations pendant l'“ après-dîner ». On est là au seuil de la mode critique naissante du salon de lecture et des conversations galantes sur les auteurs anciens ${ }^{27}$. En ce sens, le texte de Renouard témoigne des débuts du changement de paradigme de lecture que subit l'œuvre d'Ovide au Xvir siècle qui voit la transition, pour emprunter la formule de Marie-Claire Chatelain, d'un Ovide savant vers un Ovide galant ${ }^{28}$.

27 «We recognise the beginnings of a new style of discourse, deceptively facile and faintly ironic, modelled on polite social discourse. „Moss, Ovid, p. 155. Sur les débuts du salon littéraire en France, voir L. Clark-Keating, Studies on the Literary Salon in France. 1550-1615, Cambridge MA, 1941.

28 M.-C. Chatelain, Ovide savant, Ovide galant. Ovide en France dans la seconde moitié du XVII siècle, Paris, 2008. 
Le mythe de Pyrame et Thisbé a certes une composante tragique, et l'interprétation des personnages par Renouard comme des exempla d'amour tragique est en un certain sens justifiée. Mais il subsiste également dans le texte d'Ovide des aspects dissonants, voire comiques. Avant d'en venir à l'Ovide bouffon, qui incarne le « moment burlesque » d'Ovide dans l'histoire de la réception française de l'auteur, il importe de signaler que certains éléments pour ainsi dire burlesques existent déjà dans le texte d'Ovide ${ }^{29}$. Un tel cas a été relevé par Carole Newlands dans le passage de la mort de Pyrame (Ovide, Métamorphoses IV, 119-24) :

\section{[...] demisit in ilia ferrum, \\ nec mora, feruenti moriens e uulnere traxit. \\ ut iacuit resupinus humo, cruor emicat alte, \\ non aliter quam cum uitiato fistula plumbo \\ scinditur et tenui stridente foramine longas \\ eiaculatur aquas atque ictibus aera rumpit.}

Il se plonge son poignard dans le sein, que d'une main mourante il retire aussi tost de la playe toute chaude, et tombe à la renverse. Son sang bouillonnant s'eslanca en hault, tout ainsi comme lors qu'un vieil canal de plomb se creue, le petit trou qui l'est fait, fremissant darde une longue picque d'eau, qui jallit en l'air et le fend d'une extreme violence. (Trad. Renouard)

Selon Carole Newlands, la comparaison du sang qui jaillit de la blessure de Pyrame avec de l'eau qui gicle d'un tuyau endommagé introduit une touche d'humour déplacée dans un moment hautement pathétique et tragique, et elle souligne l'allusion sexuelle ${ }^{30}$. On constate d'ailleurs la manière dont Renouard, dans sa traduction, atténue la dimension érotique du texte ovidien en traduisant ilia (“l'aine ») par “sein » ${ }^{31}$. Un jet de sang qui gicle de la poitrine est bien évidemment moins sexualisé, et par là plus bienséant, que l’image originale qui évoque une éjaculation, tissant ainsi un lien entre le plaisir sexuel et la mort. Le symbolisme sexuel de la scène paraît se développer quelques vers plus loin, lorsque Thisbé aperçoit les membres tremblants de Pyrame remuer sur le sol ensanglanté (tremebunda uidet pulsare cruentum | membra solum, IV, 133-134).

29 Sur le burlesque chez Ovide, voir N. Horsfald, "Epic and Burlesque in Met. viii. 260ff. », The Classical Journal 74. 4 (1979), p. 319-332.

30 "Compounding the grotesque nature of this simile is its rather overt sexual symbolism. Pyramus' manner of dying suggests a gigantic orgasm. »C. Newlands, "The simile of the fractured pipe in Ovid's Metamorphoses 4 », Ramus 15. 2 (1986), (p. 143-153), p. 143.

31 Ceci est d'ailleurs également le choix retenu par Georges Lafaye dans sa traduction des Métamorphoses plus de trois cents ans plus tard (G. Lafaye, Ovide. Les Métamorphoses, 3 vol., Paris, 1925-1930). 


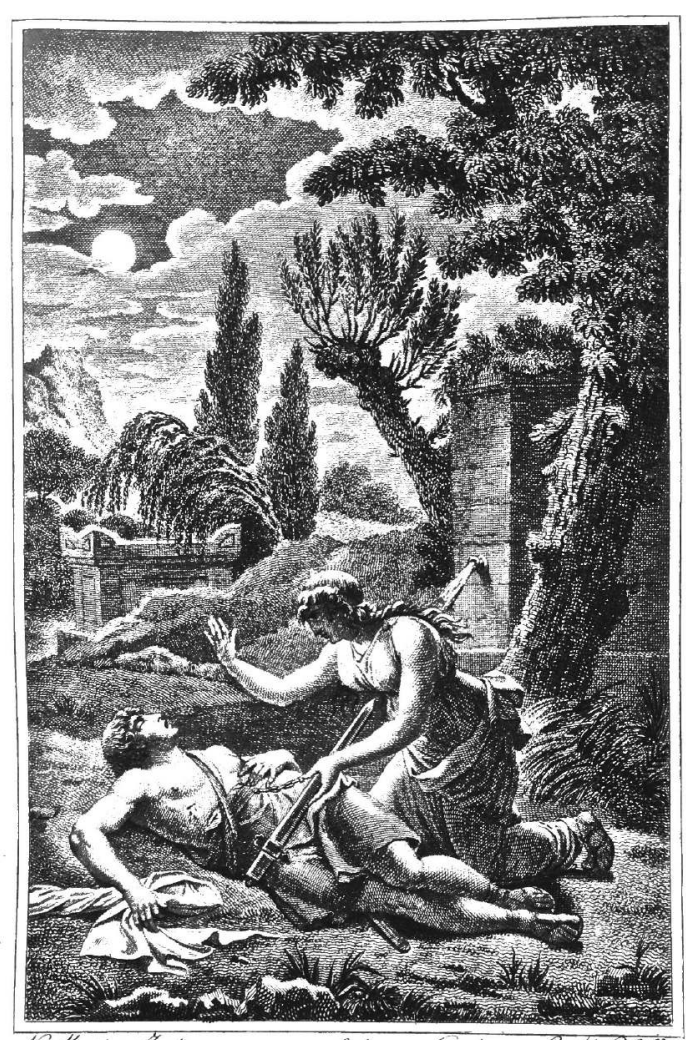

Fig. 7. Suicide de Thisbé. Métamorphoses, M.G.T. Villenave, Paris, 1806-1807. Illustration :

L. M. Halbou. Bibliothèque cantonale et universitaire de Lausanne (AVA 148/1).
Or, Renouard traduit ainsi : “ elle [Thisbé] apperceut la terre couverte de sang, \& un homme estendu, que les derniers assauts de la mort faisoient encore de battre ». En choisissant de traduire de manière métonymique le terme membrum, qui en latin peut avoir le sens de “ membre viril », par “ homme », il évacue toute possibilité de saisir l'allusion érotique. En raison de sa volonté de rendre le texte bienséant et moral, Renouard limite l'accès de son lecteur à la verve suggestive et ambiguë de la poétique ovidienne. Renouard n'intègre donc pas dans sa traduction les éléments sexuels inconvenants dans un récit tragique qui font pourtant partie intégrante du texte d'Ovide.

J'en viens maintenant au dernier texte considéré dans le cadre de cette étude : l'Ovide bouffon de Louis Richer, intitulé aussi L'Ovide travesty ou les Metamorphoses burlesques $^{32}$. Dans le sillage de la pratique traductoriale de Renouard, on peut distinguer deux courants différents de la traduction d'Ovide qui marquent la suite du siècle ${ }^{33}$ :

Après les « belles infidèles », la traduction d'Ovide évolue [...] selon deux voies qui se disjoignent, l'une qui est celle de la fidélité et va toujours plus loin dans la simplicité du style, tandis que l'autre, qui se veut davantage création littéraire, cherche à renouer avec une poétique ovidienne.

32 Ce dernier titre correspond à celui qui figure sur le frontispice (L. Richer, Ovide bouffon, Paris, 1659).

33 Chatelain, Ovide, p. 61. 
Le courant “burlesque » relève de cette seconde voie et incarne, dans la taxinomie d'Helena Taylor, la deuxième vague des traductions d'Ovide au Xvie siècle ${ }^{34}$. De manière générale, le burlesque peut se définir comme un mélange de différents genres, lexiques et registres qui a pour but de produire l'effet comique de l'hétérogène ${ }^{35}$. Je m’intéresse toutefois ici plus spécifiquement aux “ travestissements » burlesques, à savoir les “ traductions » de textes antiques. Le travestissement burlesque le plus célèbre est sans doute le Virgile travesti de Paul Scarron (publié dès 1648), qui présente une traduction parodique de l'Énéide de Virgile. Scarron est une figure importante dans le genre burlesque en France : comme le souligne Jean Serroy, « le burlesque, avant Scarron, n’était que diversion ; avec lui, il devient inversion »36. Scarron développe et accentue le « déguisement parodique » des auteurs anciens, déjà pratiqué dans la tradition du burlesque italien et, en France, par des auteurs tels que Sorel et Saint-Amant ${ }^{37}$. L'Ovide bouffon, tout comme le Virgile travesti, s'inscrit dans ce courant du burlesque qui pratique des « travestissements à fonction dévalorisante $[. .$.$] de textes canoniques$ antiques ${ }^{38}$. Cette désacralisation des textes antiques est un enjeu majeur de la Querelle des Anciens et des Modernes qui couve tout au long du XvII ${ }^{\mathrm{e}}$ siècle, et au sein de laquelle le statut de modèle littéraire des chefs-d'œuvre de l'Antiquité est profondément remis en question. Travestir Virgile, Ovide et d'autres auteurs antiques, c'est avant tout une manière pour les auteurs burlesques de s'inscrire dans ce débat littéraire et d'afficher leur adhésion au camp des Modernes ${ }^{39}$. Il s'agit en réalité moins d'une “désacralisation » de la littérature antique que d'une revendication de la liberté des auteurs à se réapproprier les classiques pour les présenter sous une nouvelle forme. L'objectif d'auteurs burlesques tels que Scarron et Richer est de faire ressortir ce que la littérature antique pouvait avoir d'intrinsèquement ridicule. Car les “ Anciens » (les auteurs antiques) sont considérés comme “ involontairement burlesques »-ils n’auraient pas conscience $\mathrm{du}$ potentiel burlesque que recèlent leurs textes - alors que le burlesque des

34 TAYlor, Lives, p. 17.

35 «Le burlesque n'utilise pas un style, mais un mélange de styles, un style mêlé, miscellaneum, qui brasse proverbes triviaux et imitations du grand style, raffinements précieux et équivoques grossières, jargon judiciaire et parisianismes à la mode, néologismes de bon ton et provincialismes archaïsants... »(C. NÉDÉLEC, “ Le Burlesque au Grand Siècle : une esthétique marginale? ”, Dix-septième siècle 224 (2004), (p. 429443), p. 433).

36 J. Serroy (éd.), Paul Scarron : Le Virgile travesti, Paris, 1988, p. 4.

37 Sur l'impact novateur de Scarron sur le genre burlesque, voir C. NÉDÉLEc, Les États et empires du burlesque, Paris, 2004, p. 97-119.

38 C. NÉDÉLEC, Burlesque, p. 267.

39 Moog-Grünewald, Metamorphosen, p. 114-119. 
“Modernes » témoigne de “lucidité critique » à l'égard des textes antiques ${ }^{40}$. Ovide est l'exception qui confirme cette règle. En effet, il joue constamment, tout particulièrement dans les Métamorphoses, avec les traditions génériques épiques pour les détourner, les subvertir, et parfois même les parodier ouvertement ${ }^{41}$.

S'il relève sans aucun doute de l'anachronisme de caractériser Ovide comme burlesque, on peut au moins relever que ses textes partagent certaines caractéristiques avec ceux de Scarron et de Richer. C'est d'ailleurs précisément l'argument que défend Richer dans l'épître dédicatoire de son Ovide bouffon, texte qui permet de saisir les mécanismes du burlesque ${ }^{42}$ :

“Pour le regard d'Ovide, je ne crois pas luy faire de tort de traiter en Burlesque un sujet qui n'a rien de serieux que dans l'esprit de nos Mythologistes qui mettent toute leur étude à chercher un sens moral dans les pensées les plus chimériques de cet Autheur et je tiens qu'il aurait employé ce mesme stile pour débiter ses agreables rêveries, si le Burlesque eust eu le mesme crédit à Rome de son temps, qu'il a maintenant à Paris. On ne doit donc pas m'accuser de l'avoir déguisé, puis qu'en métamorphosant les Métamorphoses, je n'ay fait simplement que luy retourner son habit tout usé pour avoir passé par les mains d'une infinité d'Escoliers ».

Non seulement Richer s'écarte du courant de réception scolastique en qualifiant dédaigneusement les auteurs de ces lectures comme des “ Mythologistes » et des “Escoliers », mais il va même jusqu’à présenter Ovide comme un écrivain burlesque, car il perçoit des caractéristiques que l'on pourrait qualifier de « protoburlesques » dans l'œuvre du poète augustéen. Ainsi, Richer se défend de travestir les Métamorphoses en vers burlesques en proclamant qu'Ovide lui-même aurait eu recours à ce “ mesme stile » si celui-ci avait été d'actualité à son époque. Sur ce point précis, son entreprise semble diverger de celle de Scarron dans le Virgile travesti, car dans son poème liminaire d'hommage, Tristan l'Hermite imagine la

40 Dans les Parallèles des Anciens et des Modernes, Charles Perrault, à la suite de Gabriel Guéret, relève le ridicule des auteurs anciens et distingue deux sortes de burlesque : le " burlesque involontaire », soit celui des Anciens, et le " burlesque volontaire » et contrôlé, celui des Modernes. Cf. NÉdÉLEc, Burlesque, p. 321-235.

41 Pour citer un exemple parmi tant d'autres, voir les jeux de genres dans l'épisode d'Acis, Galatée et Polyphème (Métamorphoses XIII, 722-968). Pour une analyse détaillée du dialogue entre les genres, voir J. Farrell, “ Dialogue of Genres in Ovid's 'Lovesong of Polyphemus' (Metamorphoses 13. 719-897) ", The American Journal of Philology 113. 2 (1993), p. 235-268.

42 “Epistre au Duc de Saint Aignan », dans L. Richer, Ovide bouffon, référence non paginée. 
colère de Virgile s'il voyait son Énéide “En Burlesque ainsi retourné ». Si l’on en croit Tristan l'Hermite, Scarron a dû modifier (“ retourner ») le texte virgilien afin de le rendre en vers burlesques, alors que Richer, au contraire, nie avoir retourné le texte d'Ovide puisqu'il considère que celui-ci relevait déjà du burlesque ${ }^{43}$. En d'autres termes, il revendique sa fidélité aux Métamorphoses, puisqu'il les lit comme un texte burlesque. Mais Richer va encore plus loin : il suggère que c'est sa “ traduction " qui est la plus fidèle à l'esprit d'Ovide, bien plus que celle des “ Escoliers » - c'est-à-dire celle issue de la tradition scolastique humaniste - qui, en restant prétendument proche du texte d'Ovide, trahit en réalité son esprit. Car c'est bien comme une traduction que Richer considère son propre texte, ou, pour convoquer la métaphore de Richer lui-même, comme une « métamorphose » ( en métamorphosant les Métamorphoses »). Voilà une autre manière de demeurer proche du texte d'Ovide, semble affirmer Richer implicitement, que d'emprunter le sujet de son œuvre et de l'appliquer à sa propre conception de la traduction. Considérer ce qui peut paraître une réécriture burlesque comme une traduction n'est pas absurde, puisque, comme l'affirme Jean Serroy, « les travestissements burlesques, sous leur apparent esprit de contradiction, traduisent en fait de la façon la plus fidèle l'ordonnance d'un panthéon littéraire »"44.

Alors que Renouard cherchait à éviter ou à atténuer les éléments choquants du texte ovidien, Richer, au contraire, tend justement à les faire ressortir davantage. Chez Richer, Pyrame se transperce avec son épée « tout droit entre $\mid$ Le nombril et le petit ventre, | Autres disent dans le sachet | Qui prend au deffaut du brechet ", et le jet de sang est comparé à du vin qui jaillit d'un tonneau percé45. Si le détail accordé à l'emplacement précis de la blessure de Pyrame est amplifié par rapport au texte latin - qui dit simplement in ilia (IV, 119) - et a un caractère grotesque, on constate néanmoins que Richer n'a pas repris la nature sexuelle de la mort de Pyrame. En revanche, il introduit certaines innovations qui confèrent à son texte un aspect licencieux là où le latin n'en contient pas : ainsi le mur qui sépare les amants “ Receloit certaine creuasse | Où l'on eust pû fourrer le poin, | Ou quelqu'autre chose au besoin ». Une autre innovation, cette fois-ci dans la scène

43 Sur ce point, voir TAYLOR, Lives, p. 67-68. “ On no occasion in the burlesque editions of Virgil's poetry are there any suggestions that this is what Virgil would have wanted, that this is how he would have written if he were a contemporary or that his poetry itself has inspired the changes made by his translator. This distinction between Ovidian and Virgilian burlesque is a way of representing Ovid's own parody of the tradition Virgil represented. In this respect, the identification between Ovid and the burlesque becomes more precise as it centres on a construction of Ovid's own spirit of parody. »

44 J. Serroy, Virgile, p. 1.

45 “ De ce rude coup de lancette | Un ject de sang sort \& craquette, | Comme d'un muid de vin clairet | Fraischement percé du foiret ». 
de la mort de Thisbé, permet à Richer de s'en prendre de manière enjouée aux traductions qui censurent délibérément les incongruités du texte d'Ovide ${ }^{46}$ :

Sur le garçon tomba la fille,

Mais si quelque fat en babille,

Disant comme estant son Epoux

Qu'elle devoit estre dessous,

Laissons-luy faire sa censure.

On reconnaît aisément, derrière l'expression « fat en babille », les « Mythologistes » et « Escoliers » que raillait Richer dans l'épître dédicatoire. La traduction de l'épisode de Narcisse permet de rentrer davantage en contact avec la poétique de Richer. Si le texte ovidien est d'une grande richesse poétique et mobilise des allusions à la poésie élégiaque pour symboliser la mort de $\operatorname{Narcisse}^{47}$, Richer " concrétise » la mort du jeune homme en modifiant la cause de sa mort et en fondant sa description sur le bas corporel. Chez Richer, à force d'ingurgiter l'eau de la fontaine dans laquelle il voit son reflet, Narcisse explose ${ }^{48}$. Mais l'intérêt particulier de ce passage réside dans une intervention du narrateur, qui justifie sa " traduction » radicale du texte :

J'entens bien qu'Ovide en furie

M'accuse icy de menterie,

Mais n'en déplaise au bon Autheur,

Si je suis mauvais Traducteur,

Mourir d'amour est une fable ;

Et bien que la douleur accable,

On est pour en bien discourir

46 Le texte latin dit seulement que Thisbé « se coucha sur l'épée qui était encore tiède du sang [de Pyrame] » (incubuit ferro, quod adhuc a caede tepebat, IV, 162).

47 Dans la version ovidienne (Métamorphoses III, 480-493), Narcisse, ne pouvant plus supporter son malheur, se frappe le torse (marmoreis percussit pectora palmis, 481 ; « il frappa sa poitrine avec ses mains de marbre ») et finit par succomber. Le corps mourant de Narcisse est comparé à de la cire qui fond et devient liquide 'ut intabescere flauae | igne leui cerae matutinaeque pruinae | sole tepente solent, 487-489 ; " comme fond la cire jaune auprès d'un feu léger, ou le givre matinal sous la tiédeur du soleil ») et le passage contient plusieurs échos élégiaques (voir P. E. KNox, "The Transformation of Elegy ", in Ovid's Metamorphoses and the Traditions of Augustan Poetry, Cambridge, 1986, (p. 9-26), p. 22).

48 “ Et comme une beste sauvage, | Il se met le mufle dans l'eau, | Et trinque si bien \& si beau, | Pensant tarir cette fontaine, | Qu'à force d'enfler sa bedaine, | Et faute de la décharger, | Par trop boire, \& ne point manger, | Trippe \& boudins par reverence, | Luy sortirent hors de la pance. » 
Bien malade avant que mourir.

Passons donc, Ovide \& Virgile

Ne sont pas tous mots d'Evangile.

Richer entérine ici la distance qu'il prend vis-à-vis du texte original et imagine le reproche que pourrait lui faire Ovide qui, s'il était en mesure de lire ce texte, accuserait son auteur de « menterie » dans son traitement de la mort de Narcisse. Mais bien loin de s'en excuser, il revendique au contraire son indépendance par rapport au texte latin, et renverse, une nouvelle fois, le reproche dont il est victime : c'est Ovide qui, implicitement, devient coupable de «menterie ». En effet, en affirmant que « mourir d'amour est une fable » (fable revêtant ici son sens étymologique, c'est-à-dire mensonge), Richer taxe Ovide d'invraisemblance, ce qui constitue, on le sait, un chef d'accusation sérieux dans le contexte littéraire du $\mathrm{XvII}^{\mathrm{e}}$ siècle. Le registre du bas corporel sert ainsi à donner une explication plausible et vraisemblable (“ pour en bien discourir ») à la mort de Narcisse. On peut aussi interpréter ce passage comme une manière détournée de se défendre contre ceux qui pourraient le qualifier de "mauvais Traducteur », titre que Richer récuse, d'ailleurs, dans le “Prélude» au livre III ${ }^{49}$. Notons également que “ Traducteur » est à la rime avec " Autheur », ce qui est loin d'être anodin : les frontières entre les deux pratiques sont floues au xvII ${ }^{\mathrm{e}}$ siècle ${ }^{50}$, et la dimension interprétative de la traduction est un trait qui se perçoit déjà chez les humanistes ${ }^{51}$. Mais ce sont les deux derniers vers qui sont les plus intéressants : Richer revendique son droit de ne pas prendre les auteurs anciens à la lettre parce qu'ils ne sont pas des textes sacrés, et n'ont donc pas valeur de vérité. Il critique ici la méthode de lecture des allégoristes qui ne lisent les auteurs anciens que pour y trouver un sens chrétien, un “ vrai » sens, et la référence à Virgile ne fait que renforcer l'allusion au courant allégorique, puisque Ovide et Virgile sont ensemble les deux auteurs les plus « moralisés » du Moyen Âge. En reprochant à Ovide son invraisemblance et en proposant une version burlesque plus vraisemblable de la fable, Richer ne fait que mettre le doigt sur une pratique de lecture dépassée, et ridiculise les “ Mythologistes » qui en sont coupables. Les fables d'Ovide sont précisément cela :

49 Richer ne souhaite pas être considéré “ comme Traducteur d'un Autheur illustre », mais " comme Introducteur d'un Bouffon qu'[il a] tasché de rendre agréable en ses discours » (cf. NÉDÉLEc, Burlesque, p. 319). On retrouve ici la notion du «burlesque involontaire » des Anciens.

50 Sur cette question, voir R. Zuber, Les Belles infidèles. La formation du goût classique, Paris, 1968.

51 M. JeAnneret, Le Défi des signes. Rabelais et la crise de l'interprétation à la Renaissance, Orléans, 1994, p. 28. Voir aussi M. Reynolds, The Poetry of Translation. From Chaucer and Petrarch to Homer and Logue, Oxford, 2011, p. 21-26. 
des fables, des récits fictifs qu'il ne faut pas essayer de rationaliser ni d'expliquer selon un “ plus hault sens », mais qu'il convient simplement d'apprécier pour leur sens premier. On pourrait qualifier ce passage de «méta-burlesque », puisque Richer, après avoir parodié le texte d'Ovide en vers burlesques, s'en prend aux moralistes qui cherchent à lire les Anciens comme ils liraient l'Évangile. Cela rentre dans le cadre du programme annoncé dans l'épître dédicatoire, à savoir que Richer se présente comme un traducteur en rupture avec la tradition cléricale et scolastique, et néanmoins des plus fidèles au texte latin.

Le conservatisme de l'édition de Renouard indique qu'au début du xvıI ${ }^{\mathrm{e}}$ siècle, la réception vernaculaire d'Ovide, c'est-à-dire celle qui se trouve en marge de l'étude strictement philologique de l'auteur, est encore fortement imprégnée de la tradition humaniste et scolastique. Les illustrations qui accompagnent la traduction suggèrent que cet héritage remonte encore plus loin, à l'époque des lectures chrétiennes. La tradition picturale liée au mythe de Pyrame et Thisbé expose des continuités iconographiques saisissantes qui prennent leur origine dans les enluminures médiévales et parcourent l'histoire des éditions illustrées des Métamorphoses. Le contraste avec l'Ovide bouffon de Louis Richer est prononcé : non seulement celui-ci est émancipé des courants de lecture allégorique et scolastique, mais il les tourne en dérision et les rejette au profit d'une nouvelle appropriation d'Ovide. Certes, le courant burlesque n'est en vogue que pendant une période relativement restreinte, particulièrement concentrée autour des années de la Fronde (1648-1653) ; mais elle laisse néanmoins une trace prononcée sur l'histoire littéraire française ${ }^{52}$, et malgré sa marginalité, l'Ovide bouffon s'inscrit pleinement dans ce courant qui a contribué à bouleverser le rapport aux textes antiques. Par ailleurs, le texte de Richer préfigure d'une certaine manière l'avènement du courant de lecture galant des œuvres d'Ovide qui émerge dans le dernier tiers du siècle, et peut-être même, plus généralement, l'assimilation entièrement laïque de ces textes qui marque le seuil de la réception contemporaine.

\author{
Basil Nelis \\ Wolfson College \\ Linton Road \\ Royaume-Uni, Oxford OX2 6UD \\ basil.nelis@classics.ox.ac.uk
}

52 Encore au $\mathrm{xix}^{\mathrm{e}}$ siècle, par exemple, Sainte-Beuve dénonçait le burlesque comme " cette lèpre des années de la Fronde »; Sainte-Beuve, Port-Royal, [1840-1859], éd. par M. Leroy, 1955, t. 3, p. 436-437. Cité dans Nédélec, Grand siècle, p. 432, où Nédélec montre l'étendue « transgénérique » du burlesque. 
ANABASES

Traditions et Réceptions de l'Antiquité

Revue de l'équipe de recherche E.R.A.S.M.E.

Université Toulouse-Jean Jaurès (UT2J)

NoRMES RÉDACTIONNELLES

ANABASES publie des articles dans cinq langues : français, anglais, allemand, italien et espagnol.

Les articles ne dépasseront pas 35000 signes et seront conformes aux normes de la revue,

disponibles sur le site web : http://plh.univ-tlse2.fr

Les articles seront pourvus d'un résumé en français et en anglais, ainsi que de six à huit mots-clés dans ces deux langues.

Les articles pourront être accompagnés de planches en noir et blanc.

Les comptes rendus compteront de 4500 à 6000 signes.

Site web avec Présentation, Sommaire de tous les Numéros et Bulletin d’Abonnement

http://plh.univ-tlse2.fr

Revues.org : http://anabases.revues.org

\section{Courrier}

Pour les articles :

Clément Bur (clement.bur@univ-jfc.fr)

Catherine VALENTI (catherine.valenti@univ-tlse2.fr)

Pour les comptes rendus :

NoémieVILlacÈQue (noemie.villaceque@univ-reims.fr)

Université Toulouse-Jean Jaurès (UT2J)

Équipe P.L.H. - E.R.A.S.M.E. (EA4601)

Maison de la recherche (MdR)

5, allées Antonio Machado

F-31058 Toulouse Cedex 9

Tél. : 0033/(0)5.61.50.25.56 et 57

Fax : 0033/(0)5.61.50.24.90 


\section{Historiographie et identités culturelles}

Carole QuATRELIVRe, Le sanctuaire gaulois de Gournay-sur-Aronde (Oise). Retour sur une découverte exceptionnelle des années 1970

\section{Traditions du patrimoine antique}

Christophe CorbIER, Le pindarisme et l'archéologie musicale : style, valeur et authenticité de la première Pythique à l'époque moderne

Arnaud AmiLien, Hélène en Égypte : Hérodote en dialogue avec l'épopée

\section{Archéologie des savoirs}

Sébastien CAzalas, Au jardin des exempla. Rhétorique et stratégie de l'exemplum antique dans l'œuvre politique de Jean Juvénal des Ursins (1388-1473)

Dossier - Perpétuer Ovide : aspects moraux, éditoriaux, linguistiques et culturels $\left(\mathrm{xIV}^{\mathrm{e}}-\mathrm{XvIII}{ }^{\mathrm{e}} \mathrm{s}\right.$.)

Francesca DelL'Oro, Introduction

Hélène Casanova-Robin, L'audace châtiée : Phaéton, Actéon et Icare dans la tradition latine jusqu'à la Renaissance, tours et détours d'un symbolisme

Dylan Bovet, Le commentaire latin des Métamorphoses d'Ovide : pratiques humanistes et évolutions de Regius-Micyllus (1543) à Burmann-Heinsius (I727)

Martine Furno, Ovide en classe, ou un auteur en éclats

Basil Neurs, D'un Ovide chrétien à un Ovide burlesque, du Moyen Âge au Grand Siècle : continuités et changements dans la traduction et dans l'illustration des Métamorphoses perçus à travers deux éditions du xvir siècle

Olivier ThÉvenaz, Épilogue

\section{Actualités et débats}

Tiphaine-Annabelle BESNARD, 2019 : l'année pop des musées d'archéologie. Retour sur l'exposition romaine Il classico si fa pop. Di scavi, copie e altri pasticci

\section{Relire les classiques des sciences de l'Antiquité}

Jean-Pierre AıBERT, Le premier Detienne : une relecture de « La notion mythique d'A $\lambda \hat{\eta} \theta \varepsilon\llcorner\alpha$ » (REG, 1960, p. 27-35)

Marcel DetIENNE, La notion mythique d'A $\lambda \hat{\eta} \theta \varepsilon \varepsilon \alpha$

\section{L'atelier de l'histoire : chantiers historiographiques}

Antiquités numériques (coordonné par Élodie Guillon) (1)

Élodie GuILLON, Introduction

Jaime ALvar, Le projet EPIDI : Epítetos divinos. Experiencia religiosay relaciones de poder en Hispania

Les mots de l'Antiquité (coordonné par Magali Soulatges) (10)

Jack Tномаs, L’Antiquité dans les toponymes de l’État de New York

Actualité du théâtre (coordonné par Malika Bastin-Hammou) (4)

Mathieu Ferrand, “Avons-nous perdu le Soleil ? / Ou l'avons-nous chassé ? ”

Thyeste de Sénèque, traduit par Florence Dupont. Mise en scène de

Thomas Jolly (Avignon, 2018).

Voyages et Voyageurs (coordonné parVéronique Krings) (11)

Claude Azıza, Freud à Pompéi

\section{Comptes rendus de lecture}

